\title{
The Solution of the 0-1 Multi-objective Knapsack Problem with the Assistance of Multi-objective Evolutionary Algorithms Based on Decomposition: A Comparative Study
}

\author{
Konstantinos Metaxiotis ${ }^{a}$, Konstantinos Liagkouras ${ }^{b}$
}

Decision Support Systems Laboratory, Department of Informatics, University of Piraeus, 80, Karaoli \& Dimitriou Str., 18534 Piraeus, Greece

a kmetax@unipi.gr, ${ }^{\mathrm{b}}$ kliagk@unipi.gr

Keywords: 0/1 Knapsack problem, Multi-objective optimization, Evolutionary algorithms, MOEAs

\begin{abstract}
The 0/1 knapsack problem has been studied extensively due to its practical importance in many and diverse domains such as economics, engineering and management. This paper examines the 0/1 Multiobjective Knapsack problem (MOKP) with the assistance of two state-of-the-art Multiobjective Evolutionary Algorithms (MOEAs) based on decomposition, namely the MOEA/D and the MOEA/D-DRA. The compared multiobjective algorithms are analyzed and tested on four test instances of the MOKP with the assistance of two performance metrics. Experimental results show that the two algorithms generate comparable results for the four benchmark multiobjective knapsack problems examined in this study.
\end{abstract}

\section{Introduction}

The 0/1 knapsack problem has been studied extensively due to its practical significance. Indeed many real world applications [1]-[3] from the field of finance, engineering, logistics, resource allocation and production scheduling stem from the principles of the knapsack problem. Briefly, the 0/1 knapsack problem involves selecting the most profitable items among the available pool of items, given the limited capacity of the knapsack. Analytically the classical 0/1 knapsack problem can be formulated as follows [4].

Consider a set of $n$ items represented with the assistance of a vector, $\mathrm{x}=\left(x_{1}, x_{2}, . ., x_{n}\right)$, with $x_{j}=1$ if item $j$ is included in the knapsack and $x_{j}=0$ otherwise. Each item has a profit $p_{j}$ and a weight $w_{j}$. The problem is to select a subset of the available items such that the total profit is maximized and the capacity $c$ of the knapsack is not exceeded. Formally the problem can be written as:

$$
\begin{aligned}
& \max \sum_{j=1}^{n} p_{j} x_{j} \\
& \text { subject to } \quad \sum_{j=1}^{n} w_{j} x_{j} \leq c \\
& x_{j} \in\{0,1\}, \quad j=\{1, \ldots, n\} .
\end{aligned}
$$

Many algorithms have been proposed over the last decade for both single and multiobjective knapsack problems. In this paper, we examine the 0/1 Multiobjective Knapsack problem (MOKP) with the assistance of two state-of-the-art Multiobjective Evolutionary Algorithms (MOEAs) based on decomposition, namely the Multiobjective Evolutionary Algorithm based on Decomposition (MOEA/D) [5] and the MOEA/D with Dynamical Resource Allocation (MOEA/D-DRA) [6]. The majority of the MOEAs are Pareto dominance based, meaning that the fitness of each individual at each generation is determined by its Pareto dominance relations with other solutions in the population. However, the MOEA/D and the MOEA/D-DRA make use of an aggregating function that combines the two or more objectives into a single scalar value, and in which the weights are varied in order to generate different nondominated solutions. The weights are called trade-off 
technique can be decomposed into a number of single objective optimization problems. The MOEA/D and the MOEA/D-DRA are using the decomposition principle as described above.

The remainder of the paper is organized as follows. In section 2, a description of the multiobjective 0/1 knapsack problem (MOKP) is given. In section 3 the MOEA/D and the MOEA/D-DRA are presented. The performance metrics are discussed in section 4 . In section 5 are presented the experimental results for the two algorithms with the assistance of four multiobjective 0/1 knapsack problems of Zitzler \& Thiele [4]. Finally, section 6 analyses the results and concludes the paper.

\section{Problem formulation}

The multiobjective 0/1 knapsack problem (MOKP) can be formulated as follows:

$$
\begin{aligned}
& \max f_{i}(x)=\sum_{j=1}^{n} p_{i j} x_{j}, i=1, \ldots, m \\
& \text { subject to } \sum_{j=1}^{n} w_{i j} x_{j} \leq c_{i}, i=1, \ldots, m \\
& x=\left(x_{1}, \ldots, x_{n}\right)^{T} \in\{0,1\}^{n}
\end{aligned}
$$

where $p_{i j} \geq 0$ is the profit of item $j$ in knapsack $i, w_{i j} \geq 0$ is the weight of item $j$ in knapsack $i$ and $c$ is the maximum capacity of knapsack $i$. Finally, $x_{j}=1$ if the $j^{\text {th }}$ item from the list of items is included in the knapsack.

There are two distinct approaches for handling violations related to the capacity constrain. The first approach uses a heuristic mechanism to correct any violations and the second approach penalizes solutions which violate the constraint. We opted for the second approach. In particular infeasible solutions are allowed in order to increase the diversity in the population pool; however the infeasible individuals are penalized to have less chance to be reproduced.

The penalty function takes into account the magnitude of the overall capacity constraint violation by the individual. Thus, the penalty function is expressed by the following relationship:

$$
\begin{aligned}
& \operatorname{Pen}_{i}(x)=\rho_{i}\left(\sum_{j=1}^{n} w_{i j} x_{j}-c_{i}\right) \\
& f_{i}(x)=\sum_{j=1}^{n} p_{i j} x_{j}-\operatorname{Pen}_{i}(x), \quad i=1, \ldots, m \\
& \text { where } \rho_{i}=\max _{j=1, \ldots, n}\left\{p_{i j} / w_{i j}\right\}
\end{aligned}
$$

Please notice that penalty functions are applied only to knapsacks which are over the maximum allowed capacity.

\section{Analysis of Algorithms: MOEA/D and MOEA/D-DRA}

MOEA/D. The MOEA/D uses a decomposition mechanism for converting the problem of approximation of the PF into a number of scalar optimization problems. Formally, a m-objective minimization problem can be described as:

$$
\min f(\mathbf{x})=\left(f_{1}(\mathbf{x}), f_{2}(\mathbf{x}), \ldots, f_{m}(\mathbf{x})\right)
$$


where $\mathrm{f}(\mathbf{x})$ is a m-dimentional objective vector, $\mathrm{f}_{\mathrm{i}}(\mathbf{x})$ is the $i$-th objective to be minimized and $\boldsymbol{x}$ is a decision vector. The multiobjective problem in Eq. 1 is decomposed into a number of singleobjective problems defined by a scalarizing function with different weight vectors. We consider the weighted sum and the weighted Tchebycheff as used in [5].

The weighted sum is written using the weight vector $\lambda=\left(\lambda_{1}, \lambda_{2}, \ldots, \lambda_{\mathrm{m}}\right)$, as

$$
\mathrm{g}^{\mathrm{WS}}(\mathbf{x} \mid \lambda)=\lambda_{1} \mathrm{f}_{1}(\mathbf{x})+\lambda_{2} \mathrm{f}_{2}(\mathbf{x})+\ldots,+\lambda_{\mathrm{m}} \mathrm{f}_{\mathrm{m}}(\mathbf{x}) \text {. }
$$

The weighted sum is to be minimized in its application to the multiobjective minimization problem.

The weighted Tchebycheff decomposition in [5] is written using the weight vector $\lambda$ and a reference point $\mathbf{z}^{*}=\left(\mathrm{z}_{1}^{*}, \mathrm{z}_{2}^{*}, \ldots, \mathrm{z}_{\mathrm{m}}^{*}\right)$ i.e. $\mathrm{z}_{\mathrm{i}}^{*}=\min \left\{\mathrm{f}_{\mathrm{i}}(\mathbf{x}) \mid \mathbf{x} \in \Omega\right\}$ for each $i=1, . ., m$ as the scalar optimization problems of the form:

$$
\mathrm{g}^{\mathrm{TE}}\left(\mathbf{x} \mid \boldsymbol{\lambda}, \mathbf{z}^{*}\right)=\min _{\mathrm{i}=1,2, . . \mathrm{m}}\left\{\lambda_{\mathrm{i}} \mid \mathrm{f}_{\mathrm{i}}(\mathbf{x})-\mathrm{z}_{\mathrm{i}}^{*}\right\} \text {, subject to } \mathbf{x} \in \Omega \text {. }
$$

Under certain mild conditions, in each Pareto optimal point $\mathbf{x}^{*}$, there exists a weight vector, $\lambda$ such that $\mathbf{x}^{*}$ is the optimal solution of Eq.3, where each optimal solution of Eq.3 is a Pareto optimal solution of the objective function i.e. $\min F(\mathbf{x})=\left(f_{1}(\mathbf{x}), \ldots, f_{m}(\mathbf{x})\right)^{T}$. Thus, by solving a set of single objective optimization problems defined by the Tchebycheff with different weight vectors allows the user to obtain different Pareto optimal solutions.

\section{MOEA/D-DRA}

In MOEA/D as introduced by [5], all the sub problems are treated equally and receive about the same amount of computational effort. However, a more recent study [6] assigns different levels of computational effort in each sub problem based on the different level of difficulty in obtaining the solution. In particular, the new version of MOEA/D with a dynamical resource allocation (MOEA/D-DRA) computes a utility parameter $\pi^{i}$ for each of the sub problems $i$, allowing computational effort to be distributed based on their utilities.

Below we provide a flowchart of the MOEA/D-DRA algorithm:

\section{Define: 1. Termination condition}

2. N, number of sub-problems

3. A uniform spread weight vector

4. $T$, number of weight vectors in the neighborhood of each weight vector.

\section{Initialization}

- Generate initial population by uniformly spreading and randomly sampling from search space

- Calculate the reference point for the Tchebycheff approach.

While (not equal to termination condition)

- Evaluate Objective Values

- Selection by using toumament selection method based on utility $\pi^{i}$

- Selection of mating and updating range

- Reproduction

- Repair, if element is out of boundary

- Update of solutions

$$
\begin{aligned}
& \text { If (generation is a multiplication of a pre-set value of } \mathrm{x} \text { ), } \\
& \text { - Update utility function; } \\
& \text { End } \\
& \text { End }
\end{aligned}
$$

Fig.1. Pseudo code of MOEA/D-DRA 


\section{Performance Metrics}

Hypervolume. Hypervolume, also known as S metric, is an indicator of both the convergence and diversity of an approximation set. Thus, given a set $\mathrm{S}$ containing $\mathrm{m}$ points in $\mathrm{n}$ objectives, the hypervolume of $\mathrm{S}$ is the size of the portion of objective space that is dominated by at least one point in $\mathrm{S}$. The hypervolume of $\mathrm{S}$ is calculated relative to a reference point which is worse than (or equal to) every point in $\mathrm{S}$ in every objective. The greater the hypervolume of a solution the better considered the solution.

Spread. The spread of solutions $(\Delta)$ examines whether or not the solutions span the entire Pareto optimal region [7]. Moreover, it calculates the Euclidean distance between the consecutive solutions in the obtained non-dominated set of solutions. Then it calculates the average of these distances. After that, from the obtained set of non-dominated solutions the extreme solutions are calculated. Finally, using the following metric it calculates the nonuniformity in the distribution.

$$
\Delta=\frac{\mathrm{d}_{\mathrm{f}}+\mathrm{d}_{1}+\sum_{\mathrm{i}=1}^{\mathrm{N}-1}\left|\mathrm{~d}_{\mathrm{i}}-\overline{\mathrm{d}}\right|}{\mathrm{d}_{\mathrm{f}}+\mathrm{d}_{1}+(\mathrm{N}-1) \overline{\mathrm{d}}}
$$

\section{The Test Problems}

The use four multiobjective 0/1 knapsack problems proposed by the Zitzler \& Thiele [4].Each problem has two objectives and 100, 250, 500 and 750 items. We refer to each problem as a $k-n$ problem where $k$ is the number of knapsacks (i.e. objectives) and $n$ is the number of items. Thus, the four knapsack problems are denoted as 2-100, 2-250, 2-500 and 2-750 respectively.

Table 1: Mean, Std, Median and Iqr for Hv and Spread

\begin{tabular}{|c|c|c|c|}
\hline Problem & & MOEA/D & $\begin{array}{c}\text { MOEA/D- } \\
\text { DRA }\end{array}$ \\
\hline \multirow{5}{*}{ Knapsack } & HV. Mean & $5.93 \mathrm{e}-$ & $6.17 \mathrm{e}-$ \\
and Std & $01_{3.5 \mathrm{e}-02}$ & $01_{4.1 \mathrm{e}-02}$ \\
\cline { 2 - 4 } & HV. & $5.99 \mathrm{e}-$ & $6.29 \mathrm{e}-$ \\
& Median & $01_{5.6 \mathrm{e}-02}$ & $01_{5.4 \mathrm{e}-02}$ \\
& and IQR & & \\
\cline { 2 - 4 } & SPREAD. & $6.47 \mathrm{e}-$ & $6.22 \mathrm{e}-$ \\
& Mean and & $01_{5.3 \mathrm{e}-02}$ & $01_{5.6 \mathrm{e}-02}$ \\
& Std & & \\
\cline { 2 - 4 } & SPREAD. & $6.43 \mathrm{e}-$ & $6.31 \mathrm{e}-$ \\
& Median & $01_{3.8 \mathrm{e}-02}$ & $01_{9.0 \mathrm{e}-02}$ \\
& and IQR & & \\
\hline
\end{tabular}

\begin{tabular}{|c|c|c|c|}
\hline Problem & & MOEA/D & $\begin{array}{c}\text { MOEA/D- } \\
\text { DRA }\end{array}$ \\
\hline \multirow{5}{*}{ Knapsack } & HV. Mean & $5.85 \mathrm{e}-$ & $5.73 \mathrm{e}-$ \\
2-250 & and Std & $01_{2.7 \mathrm{e}-02}$ & $01_{3.1 \mathrm{e}-02}$ \\
\cline { 2 - 4 } & HV. & $5.86 \mathrm{e}-$ & $5.78 \mathrm{e}-$ \\
& Median & $01_{4.8 \mathrm{e}-02}$ & $01_{3.4 \mathrm{e}-02}$ \\
\cline { 2 - 4 } & and IQR & & \\
\cline { 2 - 4 } & SPREAD. & $4.35 \mathrm{e}-$ & $4.54 \mathrm{e}-$ \\
& Mean and & $01_{4.9 \mathrm{e}-02}$ & $01_{6.2 \mathrm{e}-02}$ \\
\cline { 2 - 4 } & Std & & \\
\cline { 2 - 4 } & SPREAD. & $4.35 \mathrm{e}-$ & $4.40 \mathrm{e}-$ \\
\hline
\end{tabular}




\begin{tabular}{|l|c|c|c|}
\hline & $\begin{array}{c}\text { Median } \\
\text { and IQR }\end{array}$ & $01_{4.8 \mathrm{e}-02}$ & $01_{7.7 \mathrm{e}-02}$ \\
\hline
\end{tabular}

\begin{tabular}{|c|c|c|c|}
\hline Problem & & MOEA/D & $\begin{array}{l}\text { MOEA/D- } \\
\text { DRA }\end{array}$ \\
\hline \multirow{4}{*}{$\begin{array}{c}\text { Knapsack } \\
2-500\end{array}$} & $\begin{array}{c}\text { HV. Mean } \\
\text { and Std }\end{array}$ & $\begin{array}{c}5.54 \mathrm{e}- \\
01_{1.9 \mathrm{e}-02}\end{array}$ & $\begin{array}{c}5.54 \mathrm{e}- \\
01_{2.2 \mathrm{e}-02}\end{array}$ \\
\hline & $\begin{array}{c}\text { HV. } \\
\text { Median } \\
\text { and IQR }\end{array}$ & $\begin{array}{c}5.51 \mathrm{e}- \\
01_{2.8 \mathrm{e}-02}\end{array}$ & $\begin{array}{c}5.56 \mathrm{e}- \\
01_{3.5 \mathrm{e}-02}\end{array}$ \\
\hline & $\begin{array}{l}\text { SPREAD. } \\
\text { Mean and } \\
\text { Std }\end{array}$ & $\begin{array}{c}3.49 \mathrm{e}- \\
01_{3.2 \mathrm{e}-02}\end{array}$ & $\begin{array}{c}3.80 \mathrm{e}- \\
01_{6.0 \mathrm{e}-02}\end{array}$ \\
\hline & $\begin{array}{c}\text { SPREAD. } \\
\text { Median } \\
\text { and IQR }\end{array}$ & $\begin{array}{c}3.40 \mathrm{e}- \\
01_{5.3 \mathrm{e}-02}\end{array}$ & $\begin{array}{c}3.59 \mathrm{e}- \\
01_{6.3 \mathrm{e}-02}\end{array}$ \\
\hline
\end{tabular}

\begin{tabular}{|c|c|c|c|}
\hline Problem & & MOEA/D & $\begin{array}{c}\text { MOEA/D- } \\
\text { DRA }\end{array}$ \\
\hline \multirow{7}{*}{$\begin{array}{c}\text { Knapsack } \\
2-750\end{array}$} & $\begin{array}{c}\text { HV. Mean } \\
\text { and Std }\end{array}$ & $\begin{array}{c}5.52 \mathrm{e}- \\
01_{4.3 \mathrm{e}-02}\end{array}$ & $\begin{array}{c}5.35 \mathrm{e}- \\
01_{1.7 \mathrm{e}-02}\end{array}$ \\
\cline { 2 - 4 } & HV. & $5.32 \mathrm{e}-$ & $5.38 \mathrm{e}-$ \\
& Median & $01_{8.6 \mathrm{e}-02}$ & $01_{3.8 \mathrm{e}-02}$ \\
& and IQR & & \\
\cline { 2 - 4 } & SPREAD. & $7.42 \mathrm{e}-$ & $7.75 \mathrm{e}-$ \\
& $\begin{array}{c}\text { Mean and } \\
\text { Std }\end{array}$ & $01_{1.2 \mathrm{e}-01}$ & $01_{9.6 \mathrm{e}-02}$ \\
\cline { 2 - 4 } & SPREAD. & $7.73 \mathrm{e}-$ & $7.79 \mathrm{e}-$ \\
& Median & $01_{2.0 \mathrm{e}-01}$ & $01_{1.8 \mathrm{e}-01}$ \\
\hline & and IQR & & \\
\hline
\end{tabular}

Table 2: Boxplots for $\mathrm{Hv}$ and Spread
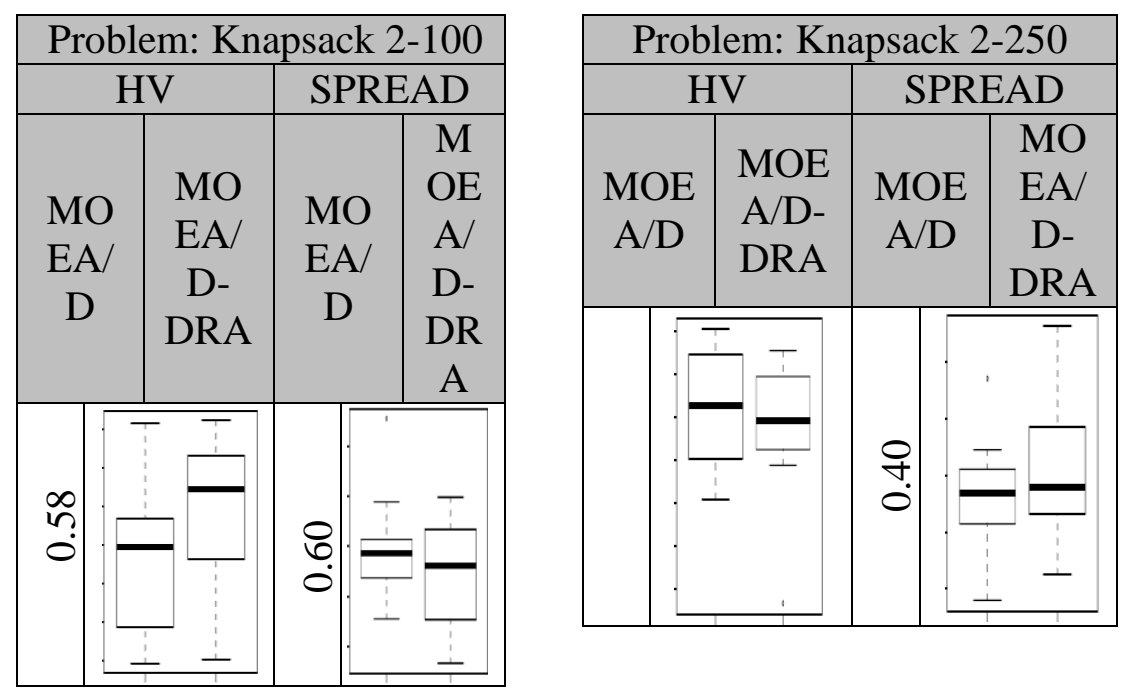

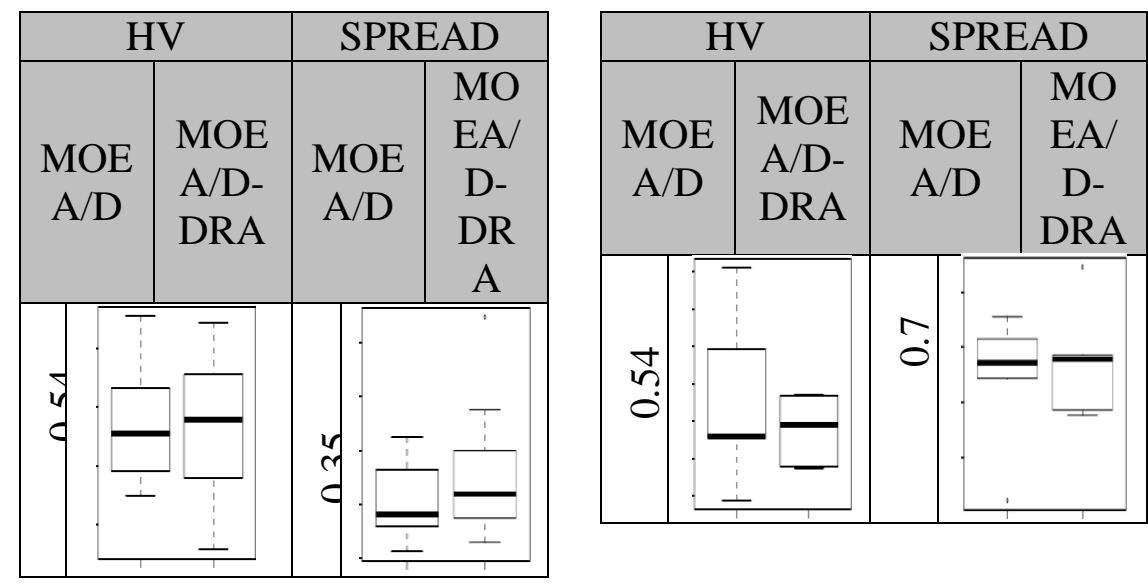

Table 1 presents the results of four bi-objective 0/1 knapsack problems proposed by the Zitzler \& Thiele. It presents the mean, standard deviation (STD), median and interquartile range (IQR) of all the independent runs carried out for Hypervolume (HV) and Spread $(\Delta)$ indicator respectively.

The higher the value of HV indicator the better the computed front. The second indicator the Spread $(\Delta)$ [7] examines the spread of solutions across the pareto front. The smaller the value of this indicator, the better the distribution of the solutions. This indicator takes a zero value for an ideal distribution of the solutions in the Pareto front.

Table 2 uses boxplots to present graphically, the performance of MOEA/D and MOEA/D-DRA respectively, for the two performance indicators, namely: HV and Spread.

\section{Analysis of the Results - Conclusions}

We applied the MOEA/D and the MOEA/D-DRA, to four multiobjective 0/1 knapsack problems proposed by the Zitzler \& Thiele [4] using the following parameter specifications:

- Coding: binary encoding,

- Population size: $N=100$,

- Termination condition: The algorithm stops after 25,000 function evaluations for each test instance.

$-T$ : the number of the weight vectors in the neighbourhood of each weight vector. $T=0.1 \mathrm{~N}$

- Number of runs for each test problem: 100 runs.

Numerical experiments have been performed using several well-known multiobjective instances of the knapsack problems provided by Zitzler \& Thiele [4]. A number of experimental comparisons with MOEA/D and MOEA/D-DRA have also been given. Experimental results show that the two algorithms generate comparable results for the four benchmark multiobjective knapsack problems examined in this study.

In particular, examining the results (Table 1 and 2) of the first indicator, the Hypervolume, we observe that the MOEA/D-DRA performs better than the MOEA/D for the 2-100 test instance Also, when examining the results regarding the Spread metric the MOEA/D-DRA performs better than the MOEA/ for the 2-100 test instance. The two algorithms generate comparable results for the 2250, 2-500 and 2-700 test instances. To conclude, from the analysis of the experimental results of this study we reach the conclusion that the MOEA/D and the MOEA/D with dynamical resource allocation (DRA) generate comparable results in terms of Hypervolume and Spread of solutions.

Future work will concentrate on testing the various approaches on a wider range of knapsack problems, with more objectives and more restricted capacities [8], [9], [10]. We also intend to experiment with different types of representations and operators. Finally, we plan to apply the knapsack problem methodology for solving a number of real world problems such as loading shipping containers given the maximum allowable weight or volume. Another practical problem would be the optimal storage of products in a distribution center given the limited space. 


\section{Acknowledgement}

Center.

The publication of this paper has been partly supported by the University of Piraeus Research

\section{References}

[1] Liagkouras, K. and Metaxiotis, K.: A new Probe Guided Mutation operator and its application for solving the cardinality constrained portfolio optimization problem, Expert Systems with Applications 41 (2014) 6274-6290.

[2] Metaxiotis, K and Liagkouras, K.: Multiobjective evolutionary algorithms for portfolio management: a comprehensive literature review, Expert Systems with Applications 39 (14), (2012) 11685-11698.

[3] Liagkouras, K. and Metaxiotis, K.: An Elitist Polynomial Mutation Operator for Improved Performance of MOEAs in Computer Networks, Computer Communications and Networks (ICCCN), 2013 22nd International Conference on, pages 1-5, (2013) IEEE.

[4] Zitzler, E. and Thiele, L.: Multiobjective Evolutionary Algorithms: A Comparative Case Study and the Strength Pareto Approach, IEEE Transactions on Evolutionary Computation 3 (1999) 257-271.

[5] Zhang, Q. and Li, H.: MOEA/D: A Multiobjective Evolutionary Algorithm Based on Decomposition. IEEE Trans. on Evolutionary Computation 11 (2007) 712-731.

[6] Zhang, Q., Liu, W. and Li, H.: The Performance of a New Version of MOEA/D on CEC09 Unconstrained MOP Test Instances." IEEE Congress on Evolutionary Computation,Trondheim, Norway, (2009).

[7] Kumar, R. and Singh, P.: Assessing solution quality of biobjective 0-1 knapsack problem using evolutionary and heuristic algorithms, Applied Soft Computing, Vol. 10, No. 3, 711-718, 2010.

[8] Wang, H., Kochenberger, G. and Glover, F.: A computational study on the quadratic knapsack problem with multiple constraints, Computers \& Operations Research, Vol. 39, No. 1, 3-11, 2012 .

[9] Zhang, J.: Comparative study of several intelligent algorithms for knapsack problem, Procedia Environmental Sciences, Vol. 11, Part A, 163-168, 2011.

[10]Kafafy A., Bounekkar A., Bonnevay S.: A hybrid evolutionary metaheuristics (HEMH) applied on 0/1 multiobjective knapsack problems. GECCO-2011: 497-504. 